\title{
Catellatospora koreensis sp. nov., a novel actinomycete isolated from a gold-mine cave
}

\author{
Soon Dong Lee, Sa-Ouk Kang and Yung Chil Hah
}

Department of

Microbiology, College of Natural Sciences and Research Center for Molecular Microbiology, Seoul National University, Seoul 151-742,

Republic of Korea
Author for correspondence: Yung Chil Hah. Tel: +822880 6700. Fax: +8228884911. e-mail: hahyungc@snu.ac.kr

\begin{abstract}
A new actinomycete strain, LM $042^{\top}$, which was isolated from a gold-mine cave in Kongju, Republic of Korea, is described by phenotypic and genotypic characters. The organism formed short chains of non-motile spores and globose bodies from substrate mycelium. An aerial mycelium was absent. This organism was chemotaxonomically characterized by the presence of mesodiaminopimelic acid, rhamnose, xylose, glucose, mannose and ribose in wholecell hydrolysates (a type II cell wall and a variant of sugar pattern D), a glycolyl type of muramic acid, DNA G+C content of $70.4 \mathrm{~mol} \%$, a type PII phospholipid pattern (phosphatidylethanolamine as a diagnostic nitrogenous phospholipid), a tetrahydrogenated menaquinone with 10 isoprene units as a major menaquinone, and fatty acid profiles predominated by iso-branched hexadecanoic acid, iso-branched pentadecanoic acid and heptadcenoic acid. A comparative analysis of $16 \mathrm{~S}$ rDNA sequences indicated that this organism formed a distinct clade within the evolutionary radiation of the family Micromonosporaceae and clustered with members of the genus Catellatospora. The 16S rDNA similarity values between the isolate and its phylogenetic neighbours, the two subspecies of Catellatospora citrea and Catellatospora tsunoense, were $95 \cdot 0-95 \cdot 2 \%$ and $94.9 \%$, respectively. An equidistant relationship was observed among the isolate, Catellatospora ferruginea and all other members of the Micromonosporaceae genera (levels of similarity $93.0-94.0 \%$ ). The combination of physiological, chemotaxonomic and DNA-DNA hybridization data supported that this organism is a novel species of the genus Catellatospora, for which the name Catellatospora
\end{abstract} koreensis sp. nov. is proposed. The type strain is LM $042^{\top}$ ( = IMSNU 50729').

Keywords: Catellatospora koreensis, soil bacteria, actinomycetes, phylogeny

\section{INTRODUCTION}

The genus Catellatospora was described by Asano \& Kawamoto (1986) for aerobic, Gram-positive and mesophilic actinomycetes which belong to the family Micromonosporaceae of the order Actinomycetales and are morphologically characterized by short chains of non-motile spores borne directly on the substrate mycelium without the formation of aerial mycelium. This genus has a type II/D cell wall (mesodiaminopimelic acid, 3-hydroxy-diaminopimelic acid, arabinose and xylose) (Lechevalier \& Lechevalier, 1970), the glycolyl type of muramic acid (Uchida \& Aida, 1984) and a type PII phospholipid pattern

The GenBank accession number for the $16 \mathrm{~S}$ rDNA sequence of strain LM $042^{\top}$ is AF171700. (phosphatidylethanolamine as a diagnostic phospholipid) (Lechevalier et al., 1981), and originally contained four species, Catellatospora citrea (with Catellatospora citrea subsp. methionotrophica), Catellatospora ferruginea, Catellatospora matsumotoense and Catellatospora tsunoense. Based on the type of a major menaquinone and the presence or absence of the cell-wall sugar, 3-O-methylrhamnose, members of the genus are divided into two groups. Catellatospora citrea with the subspecies Catellatospora citrea subsp. methionotrophica and Catellatospora tsunoense are characterized by the presence of menaquinones with nine isoprene units and the absence of 3-Omethylrhamnose, whereas Catellatospora ferruginea and Catellatospora matsumotoense have menaquinones with ten isoprene units and 3-O-methylrhamnose is present (Asano \& Kawamoto, 1986, 1988; 
Asano et al., 1989). Comparative analysis of $16 \mathrm{~S}$ rDNA sequences indicated that members of the genus Catellatospora, except for Catellatospora matsumotoense, were true members of the family Micromonosporaceae but were phylogenetically heterogeneous (Koch et al., 1996) as represented by the chemotaxonomic groupings mentioned above. It has been recently proposed that Catellatospora matsumotoense be transferred to the genus Micromonospora as $M$. matsumotoense based on its 16S rDNA sequence and phenotypic characters (Lee et al., 1999).

The family Micromonosporaceae was emended by Koch et al. (1996) based on 16S rDNA sequence studies and contains the genera Actinoplanes, Catellatospora, Catenuloplanes, Couchioplanes, Dactylosporangium and Pilimelia in addition to the type genus Micromonospora. Recently, the genera Spirilliplanes (Tamura et al., 1997) and Verrucosispora (Rheims et al., 1998) were described as new members of this family. The family Micromonosporaceae forms a coherent group in the actinobacterial phylogenetic tree and has been placed in the suborder Micromonosporineae of the order Actinomycetales (Stackebrandt et al., 1997), with description of the family-specific $16 \mathrm{~S}$ rDNA signatures. The genera of this family are readily differentiated from each other on the basis of chemotaxonomic, morphological and physiological properties (Asano \& Kawamoto, 1986; Goodfellow et al., 1990; Rheims et al., 1998; Tamura et al., 1994, 1997; Yokota et al., 1993).

A polyphasic approach based on phenotypic and genotypic classification methods is required for determining the precise taxonomic position of a new isolate. In this paper we describe the characterization and classification of isolate LM $042^{\mathrm{T}}$, and we propose that this organism should be classified as a new species of the genus Catellatospora, as Catellatospora koreensis sp. nov. Strain LM $042^{\mathrm{T}}$ has been deposited in the Culture Collection Center of the Institute of Microbiology, Seoul National University, as IMSNU $50729^{\mathrm{T}}$.

\section{METHODS}

Micro-organisms and culture conditions. Strain LM $042^{\mathrm{T}}$ was isolated from a soil collected at a gold-mine cave in Kongju, Republic of Korea. The isolation medium contained (per 1 distilled water); yeast extract (Difco), $0 \cdot 1 \mathrm{~g}$; glucose, $0 \cdot 1 \mathrm{~g} ; \mathrm{K}_{2} \mathrm{HPO}_{4}, 0.5 \mathrm{~g} ; \mathrm{Na}_{2} \mathrm{HPO}_{4}, 0.7 \mathrm{~g} ; \mathrm{KNO}_{3}, 0 \cdot 1 \mathrm{~g} ; \mathrm{NaCl}$, $0.3 \mathrm{~g} ; \mathrm{MgSO}_{4} \cdot 7 \mathrm{H}_{2} \mathrm{O}, 0.1 \mathrm{~g} ; \mathrm{CaCl}_{2} .2 \mathrm{H}_{2} \mathrm{O}, 0.02 \mathrm{~g}$ and $1 \mathrm{ml}$ trace mineral solution $\left(\mathrm{FeSO}_{4} .7 \mathrm{H}_{2} \mathrm{O}, 200 \mathrm{mg}\right.$; $\mathrm{ZnSO}_{4} .7 \mathrm{H}_{2} \mathrm{O}, \quad 180 \mathrm{mg} ; \quad \mathrm{MnSO}_{4} \cdot 4 \mathrm{H}_{2} \mathrm{O}, \quad 20 \mathrm{mg}$; $\mathrm{CuSO}_{4} .5 \mathrm{H}_{2} \mathrm{O}, 90 \mathrm{mg} ; \mathrm{CoSO}_{4} .7 \mathrm{H}_{2} \mathrm{O}, 10 \mathrm{mg} ; \mathrm{H}_{3} \mathrm{BO}_{3}, 200 \mathrm{mg}$ and $\left(\mathrm{NH}_{4}\right)_{6} \mathrm{Mo}_{7} \mathrm{O}_{24} \cdot 4 \mathrm{H}_{2} \mathrm{O}, 5 \mathrm{mg}$ in 11 distilled water). Isolate LM $042^{\mathrm{T}}$ was maintained on yeast extract/malt extract agar [medium 2 of the International Streptomyces Project (ISP medium 2)] and as mycelial fragments or spores in $20 \%(\mathrm{v} / \mathrm{v})$ glycerol or as lyophilized cells. For comparative purposes, the following type strains of the genus Catellatospora were used in this study: Catellatospora citrea subsp. citrea IFO $14495^{\mathrm{T}}$ (= IMSNU 22008 $)$, Catel- latospora citrea subsp. methionotrophica IFO $14553^{\mathrm{T}}(=$ IMSNU 22006 ${ }^{\mathrm{T}}$, Catellatospora ferruginea IFO $14496^{\mathrm{T}}(=$ IMSNU 22009 ${ }^{\mathrm{T}}$ ), Catellatospora matsumotoense IFO $14550^{\mathrm{T}}$ (= IMSNU 22003 ${ }^{\mathrm{T}}$ ) and Catellatospora tsunoense IFO $14552^{\mathrm{T}}$ (= IMSNU 22005 $5^{\mathrm{T}}$.

Morphology and pigmentation. For examining the degree of growth, pigmentation and aerial mycelia, isolate LM $042^{\mathrm{T}}$ was grown on ISP medium 2, oatmeal agar (ISP medium 3) and inorganic salts/starch agar (ISP medium 4) as described by Shirling \& Gottlieb (1966), and on glucose/asparagine agar (Waksman, 1950), yeast extract/glucose agar (Asano \& Kawamoto, 1986), Bennett agar, Hickey-Tresner agar (Waksman, 1961), yeast extract agar, nutrient agar and tapwater agar. Production of melanin pigment was examined using tyrosine agar (ISP medium 7) and peptone/yeast extract/iron agar (ISP medium 6). For scanning electron microscopy, strain LM $042^{\mathrm{T}}$ was grown on ISP medium 3 and ISP medium 4 for $21 \mathrm{~d}$. The agar blocks prepared from the growth medium were fixed with osmium tetroxide and dehydrated using a graded ethanol series. After replacing the ethanol with isoamyl acetate, the samples were criticalpoint-dried, sputter-coated with gold and observed by a Stereoscan model 260 scanning electron microscope (Cambridge).

Physiological characteristics. The ranges for temperature and $\mathrm{pH}$, and $\mathrm{NaCl}$ tolerance were determined on ISP medium 2. Degradation of adenine, hypoxanthine, DL-tyrosine and xanthine was studied as described by Gordon et al. (1974). Urease activity was determined by a colour change using Bacto urea broth (Difco). The production of hydrogen sulfide was tested using a lead acetate strip. Catalase activity was determined with a $3 \%(\mathrm{v} / \mathrm{v})$ hydrogen peroxide solution. Nitrate reductase activity was determined with an equal mixture of $0.5 \% \alpha$-naphthylamine and $0.8 \%$ sulfanilic acid. Degradation of hippurate was investigated with $12 \%$ acidified ferric chloride on nutrient broth supplemented with $1 \%$ sodium hippurate. Hydrolysis of casein, gelatin and starch was studied by previously described methods (Mac Faddin, 1980). Hydrolysis of DNA was determined using Bacto DNase test agar (Difco). Susceptibility to antibiotics and chemical inhibitors at the defined concentrations was tested using nutrient agar. Utilization of carbohydrates as sole carbon source was determined using yeast nitrogen base without amino acids (Difco) as described previously (Asano \& Kawamoto, 1986).

Chemotaxonomy. The isomer of diaminopimelic acid was determined by the method of Staneck \& Roberts (1974). The composition of whole-cell sugars was determined by GC (Saddler et al., 1991). The acyl type of muramic acid was determined by the colorimetric method of Uchida \& Aida (1984). Menaquinones and phospholipids were extracted by the integrated method of Minnikin et al. (1984). The purified menaquinones were analysed by using HPLC (Kroppenstedt, 1985) and a Quattro mass spectrometer (Micromass). The resultant phospholipids were separated by two-dimensional TLC (Minnikin et al., 1977) and identified as described by Collins et al. (1982). The presence of mycolic acids was determined by TLC (Minnikin et al., 1980). Cellular fatty acid methyl esters were prepared from cells grown on yeast extract/glucose broth at $30^{\circ} \mathrm{C}$ for $3 \mathrm{~d}$ (Minnikin, 1988) and were analysed with a Hewlett Packard model 5890A gas chromatograph equipped with a SPB-1 fused silica capillary column (Supelco) and a flameionization detector. The resultant peaks were identified with standard samples (catalogue no. 1114; Matreya). 
Table 1. Strains and $16 \mathrm{~S}$ rDNA accession numbers used in this study

IMSNU, Institute of Microbiology, Seoul National University, Korea; DSM, DSMZ - Deutsche Sammlung von Mikroorganismen und Zellkulturen, Braunschweig, Germany; IFO, Institute for Fermentation, Osaka, Japan.

\begin{tabular}{|c|c|c|}
\hline Taxon & Strain & Accession no. \\
\hline Catellatospora koreensis & LM $042^{\mathrm{T}}(=$ IMSNU 50729 $)$ & AF171700 \\
\hline Catellatospora citrea subsp. citrea & IMSNU $22008^{\mathrm{T}}$ & AF152106 \\
\hline Catellatospora citrea subsp. methionotrophica & IMSNU $22006^{\mathrm{T}}$ & AF152107 \\
\hline Catellatospora ferruginea & IMSNU $22009^{\mathrm{T}}$ & AF152108 \\
\hline Catellatospora matsumotoense & IMSNU $22003^{\mathrm{T}}$ & AF152109 \\
\hline Catellatospora tsunoense & IMSNU $22005^{\mathrm{T}}$ & AF 152110 \\
\hline Actinoplanes brasiliensis & DSM $43805^{\mathrm{T}}$ & X93185 \\
\hline Actinoplanes cyaneus & DSM $46137^{\mathrm{T}}$ & X93186 \\
\hline Actinoplanes philippinensis & DSM $43019^{\mathrm{T}}$ & X93187 \\
\hline Actinoplanes regularis & DSM $43151^{\mathrm{T}}$ & X93188 \\
\hline Couchioplanes caeruleus subsp. caeruleus & IFO $13939^{\mathrm{T}}$ & D85479 \\
\hline Catenuloplanes japonicus & IFO $14176^{\mathrm{T}}$ & D85476 \\
\hline Dactylosporangium aurantiacum & DSM $43157^{\mathrm{T}}$ & X93191 \\
\hline Dactylosporangium fulvum & DSM $43917^{\mathrm{T}}$ & X93192 \\
\hline Dactylosporangium matsuzakiensis & DSM $43810^{\mathrm{T}}$ & X93193 \\
\hline Dactylosporangium roseum & DSM $43916^{\mathrm{T}}$ & X93914 \\
\hline Dactylosporangium thailandense & DSM $43158^{\mathrm{T}}$ & X92630 \\
\hline Dactylosporangium vinaceum & DSM $32823^{\mathrm{T}}$ & X93196 \\
\hline Micromonospora aurantiaca & DSM $43813^{\mathrm{T}}$ & X92604 \\
\hline Micromonospora brunnea & DSM $43814^{\mathrm{T}}$ & X92605 \\
\hline Micromonospora carbonacea subsp. carbonacea & DSM $43168^{\mathrm{T}}$ & X92599 \\
\hline Micromonospora chalcea & DSM $43026^{\mathrm{T}}$ & X92594 \\
\hline Micromonospora chersina & DSM $44151^{\mathrm{T}}$ & X92628 \\
\hline Micromonospora coerulea & DSM $43143^{\mathrm{T}}$ & X92598 \\
\hline Micromonospora echinospora subsp. echinospora & DSM $43816^{\mathrm{T}}$ & X92607 \\
\hline Micromonospora halophytica subsp. halophytica & DSM $43171^{\mathrm{T}}$ & X92601 \\
\hline Micromonospora inositola & DSM $43819^{\mathrm{T}}$ & X92610 \\
\hline Micromonospora olivasterospora & DSM $43868^{\mathrm{T}}$ & X92613 \\
\hline Micromonospora purpurea & DSM $43036^{\mathrm{T}}$ & X92595 \\
\hline Micromonospora purpureochromogenes & DSM $43821^{\mathrm{T}}$ & X92611 \\
\hline Micromonospora rhodorangea & DSM $1039^{\mathrm{T}}$ & X92612 \\
\hline Micromonospora rosaria & DSM $803^{\mathrm{T}}$ & X92631 \\
\hline Pilimelia anulata & DSM $43039^{\mathrm{T}}$ & X93189 \\
\hline Pilimelia terevasa & DSM $43040^{\mathrm{T}}$ & X93190 \\
\hline Spirilliplanes yamanashiensis & IFO $15828^{\mathrm{T}}$ & D63912 \\
\hline Verrucosispora gifhornensis & DSM $44337^{\mathrm{T}}$ & Y15523 \\
\hline
\end{tabular}

DNA base composition and DNA-DNA hybridization. Genomic DNA was isolated as described previously (Hopwood et al., 1985). The $\mathrm{G}+\mathrm{C}$ content of the DNA was determined by HPLC (Mesbah et al., 1989). DNA-DNA hybridization studies were carried out by the previously described methods of Hopwood et al. (1985).

Phylogenetic analysis and nucleotide accession numbers. The cloning of $16 \mathrm{~S}$ rDNA following PCR-mediated amplification from genomic DNA was carried out as described previously (Lee et al., 2000). The cloned 16S rDNA sequence was determined using the Cy5 AutoRead Sequencing Kit (Pharmacia). The sequence obtained was aligned by using the program CLUSTAL W (Thompson et al., 1994) with those of the type strains of the family Micromonosporaceae and related actinomycete taxa. Evolutionary distance matrices were calculated by using the method of Jukes \& Cantor (1969), and a phylogenetic tree was reconstructed by the neighbour-joining method (Saito \& Nei, 1987). The reliability of the tree topology was evaluated by using SEQBOOT, DNADIST, NEIGHBOR and CONSENSE programs in the PHYLIP package (Felsenstein, 1985, 1993), based on 1000 bootstrapped trees generated.

The reference sequences used in this study are listed in Table 1. The strains used as outgroup members were Nocardia asteroides DSM 43757 ${ }^{\mathrm{T}}$ (X80606), Saccharomonospora viridis NCIMB $9602^{\mathrm{T}}$ (Z38021), Streptomyces griseus KCTC $9080^{\mathrm{T}}(\mathrm{M} 76388)$ and Streptosporangium roseum DSM $43021^{\mathrm{T}}$ (X89947). The 16S rDNA sequence of isolate LM $042^{\mathrm{T}}(=$ 
IMSNU 50729 ) has been deposited in the GenBank database under accession number AF171700.

\section{RESULTS}

\section{Morphology}

Isolate $\mathrm{LM} 042^{\mathrm{T}}$ produced a well-developed and branched substrate mycelium. An aerial mycelium was not formed on any media tested. Straight, relatively short chains of non-motile spores were borne directly on substrate mycelium. Each spore was cylindrical and its surface was smooth (Fig. 1a). The substrate mycelium was cream-coloured to light yellow. Globose bodies, which were morphologically similar to the globose bodies of Catellatospora tsunoense (Asano et al., 1989), were also observed abundantly on oatmeal agar by strain LM $042^{\mathrm{T}}$ (Fig. 1b).

\section{Chemotaxonomic characteristics}

Isolate LM $042^{\mathrm{T}}$ contained meso-diaminopimelic acid, rhamnose, xylose, glucose, mannose and ribose in whole-cell hydrolysates, indicating that this organism
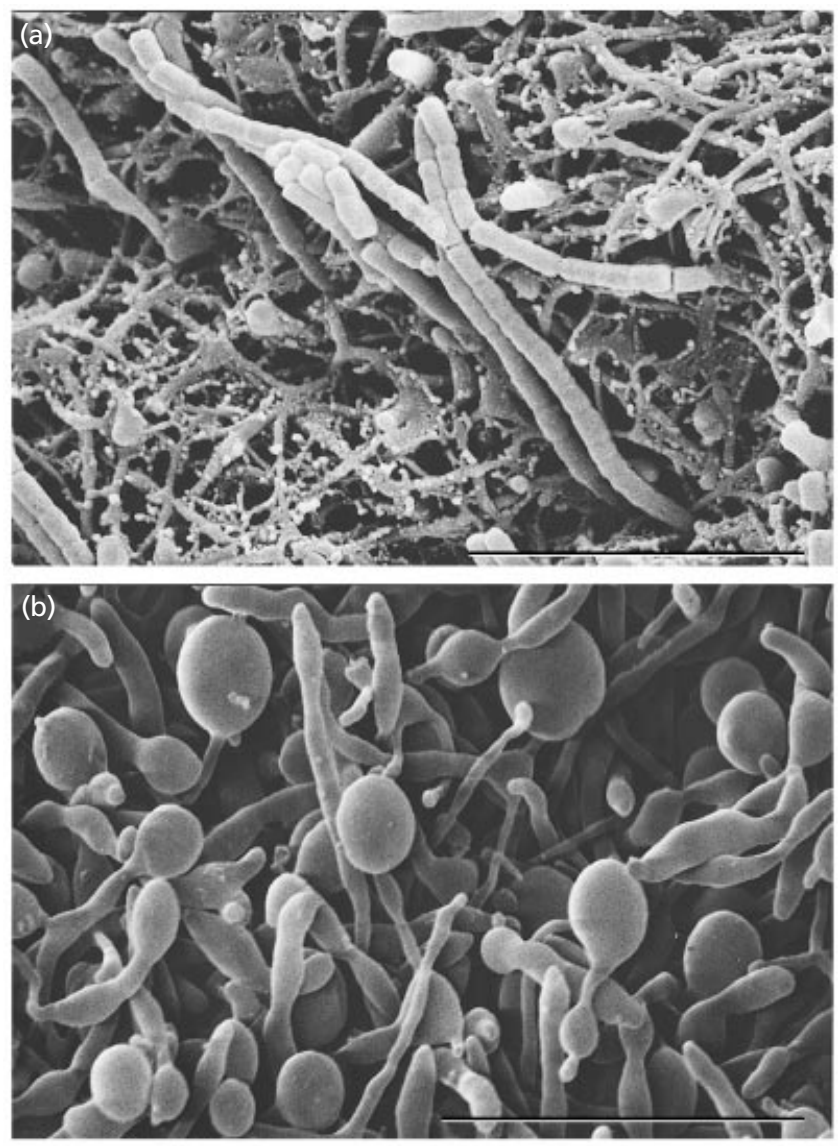

Fig. 1. Scanning electron micrograph of isolate $L M 042^{\top}$ grown on agar media for $21 \mathrm{~d}$. (a) Spore chains directly formed from substrate mycelium on oatmeal agar (ISP medium 3). (b) Globose bodies on inorganic salts/starch agar (ISP medium 4). Bars, $5 \mu \mathrm{m}$. has a type II cell wall and a variant of sugar pattern D (Lechevalier \& Lechevalier, 1970). The acyl type of muramic acid in the cell wall was glycolyl. Mycolic acids were not detected. The polar lipids contained phosphatidylethanolamine, diphosphatidylglycerol and phosphatidylinositol (a type PII phospholipid pattern of Lechevalier et al., 1981). Glucosaminecontaining phospholipid and phosphatidylcholine were not detected. The $\mathrm{G}+\mathrm{C}$ content of the DNA was $70.4 \mathrm{~mol} \%$. (Table 2). The major menaquinone of isolate $\mathrm{LM} 042^{\mathrm{T}}$ was $\mathrm{MK}-10\left(\mathrm{H}_{4}\right)$; in addition, small amounts of MK-10( $\left.\mathrm{H}_{6}\right)$ and MK-9 $\left(\mathrm{H}_{4}\right)$ were also present. It has been previously reported that members of the genus Catellatospora contain major menaquinones, MK-9 $\left(\mathrm{H}_{4}\right)$ or MK-10 $\left(\mathrm{H}_{6}, \mathrm{H}_{8}\right)$ depending on the strain (Asano \& Kawamoto, 1986, 1988; Asano et al., 1989). All of the data available for the type strains of the genus Catellatospora were also examined. The data obtained in this study, except for the menaquinone composition of Catellatospora tsunoense, were in agreement with the data published previously. It was shown that the major menaquinone of Catellatospora tsunoense was identical to that of the isolate LM $042^{\mathrm{T}}$ in our analysis (Table 2). The predominant fatty acids of isolate LM $042^{\mathrm{T}}$ were iso-branched hexadecanoic acid (iso- $\mathrm{C}_{16: 0}$ ), iso-pentadecanoic acid (iso- $\left.\mathrm{C}_{15: 0}\right)$ and heptadecenoic acid $\left(\mathrm{C}_{17: 1}\right)$. A significant amount of saturated, unsaturated and anteisobranched fatty acids were also present, whereas 10 -methyl-branched fatty acid was not detected. The cellular fatty acid compositions of isolate LM $042^{\mathrm{T}}$ and Catellatospora reference strains are shown in Table 3.

\section{Physiological characteristics}

Isolate LM $042^{\mathrm{T}}$ grew strictly aerobically. This organism showed catalase and nitrate reductase activity but not urease activity. $\mathrm{H}_{2} \mathrm{~S}$ production was not observed. The ranges of temperature and $\mathrm{pH}$ for growth were $20-37^{\circ} \mathrm{C}$ and $\mathrm{pH} 6 \cdot 0-9 \cdot 0$, respectively. Growth did not occur at $42{ }^{\circ} \mathrm{C}$ and in the presence of $2 \% \mathrm{NaCl}$. No soluble pigments were produced in any of the media tested. Strain LM $042^{\mathrm{T}}$ exhibited good growth on oatmeal agar, inorganic salts/starch agar, glucose/asparagine agar, yeast extract/glucose agar, Hickey-Tresner agar and Bennett agar. However, growth of this organism was poor to moderate on glycerol/asparagine agar, yeast extract agar, nutrient agar, tyrosine agar and tap-water agar. The physiological characteristics differentiating isolate LM $042^{\mathrm{T}}$ from the reference strains of the genus Catellatospora are shown in Table 4.

\section{Phylogenetic analysis}

The almost complete 16S rDNA sequence of isolate LM $042^{\mathrm{T}}$, consisting of a continuous stretch of 1510 nucleotides $(97 \%$ of the Escherichia coli sequence; Brosius et al., 1978), was determined following cloning and sequencing of the amplified gene. The 
Table 2. Chemotaxonomic characteristics of isolate $L M 042^{\top}$ and the reference strains of the genus Catellatospora

Abbreviations: meso-DAP, meso-diaminopimelic acid; 3-OH-DAP, 3-hydroxy-diaminopimelic acid; Xyl, xylose; Ara, arabinose; Gal, galactose; DPG, diphosphatidylglycerol; PE, phosphatidylethanolamine; PG, phosphatidylglycerol; PI, phosphatidylinositol; PIM, phosphatidylinositol mannoside; PME, phosphatidylmethylethanolamine; PL, unknown phospholipid(s). Abbreviations for menaquinones are exemplified by MK-10 $\left(\mathrm{H}_{4}\right)$ : a tetrahydrogenated menaquinone with ten isoprene units.

\begin{tabular}{|c|c|c|c|c|c|}
\hline Taxon & $\begin{array}{c}\text { Cell wall } \\
\text { diamino } \\
\operatorname{acid}(\mathbf{s})\end{array}$ & $\begin{array}{l}\text { Characteristic } \\
\text { sugar(s) }\end{array}$ & Polar lipids & $\begin{array}{c}\text { Major } \\
\text { menaquinone(s) }\end{array}$ & $\begin{array}{c}G+C \\
\text { content } \\
(\mathrm{mol} \%)\end{array}$ \\
\hline Isolate LM $042^{\mathrm{T}}$ & meso-DAP & Xyl & DPG, PI, PE & $\mathrm{MK}-10\left(\mathrm{H}_{4}\right)$ & $70 \cdot 4$ \\
\hline $\begin{array}{l}\text { Catellatospora tsunoense IMSNU } \\
22005^{\mathrm{T}}\end{array}$ & meso-DAP, 3-OH-DAP & Ara, Xyl, Gal & $\begin{array}{l}\text { DPG, PI, PG, } \\
\text { PME }\end{array}$ & $\mathrm{MK}-10\left(\mathrm{H}_{4}\right)$ & $70 \cdot 6$ \\
\hline $\begin{array}{l}\text { Catellatospora citrea subsp. citrea } \\
\text { IMSNU } 22008^{\mathrm{T}}\end{array}$ & meso-DAP, 3-OH-DAP & Ara, Xyl, Gal & $\begin{array}{l}\text { DPG, PI, PIM, } \\
\text { PE, PG, PL }\end{array}$ & MK-9 $\left(\mathrm{H}_{4}, \mathrm{H}_{6}\right)$ & $71 \cdot 5$ \\
\hline $\begin{array}{l}\text { Catellatospora citrea subsp. } \\
\text { methionotrophica IMSNU } 22006^{\mathrm{T}}\end{array}$ & meso-DAP, 3-OH-DAP & Ara, Xyl, Gal & DPG, PI, PE & MK-9 $\left(\mathrm{H}_{4}, \mathrm{H}_{6}\right)$ & $70 \cdot 8$ \\
\hline $\begin{array}{l}\text { Catellatospora ferruginea IMSNU } \\
22009^{\mathrm{T}} \dagger\end{array}$ & meso-DAP, 3-OH-DAP & Ara, Xyl, Gal & $\begin{array}{l}\text { DPG, PI, PIM, } \\
\text { PE, PG, PL }\end{array}$ & $\begin{array}{l}\text { MK-10 } \\
\left(\mathrm{H}_{6}, \mathrm{H}_{8}\right)\end{array}$ & $71 \cdot 5$ \\
\hline $\begin{array}{l}\text { Catellatospora matsumotoense } \\
\text { IMSNU } 22003^{\mathrm{T} * \dagger}\end{array}$ & meso-DAP, 3-OH-DAP & Ara, Xyl, Gal & $\begin{array}{l}\text { DPG,PI, PE, PG, } \\
\text { PL }\end{array}$ & $\begin{array}{l}\mathrm{MK}-10 \\
\left(\mathrm{H}_{6}, \mathrm{H}_{8}\right)\end{array}$ & $71 \cdot 0$ \\
\hline
\end{tabular}

* Contains 3-methylrhamnose as an additional diagnostic sugar.

$\dagger$ According to Asano et al. (1989) and Lee et al. (1999).

Table 3. Cellular fatty acid compositions (\%) of isolate LM $042^{\top}$ and Catellatospora reference strains

Values less than $1 \%$ not shown. Abbreviations: i, iso; ai, anteiso; 10Me, 10-methyl.

\begin{tabular}{|c|c|c|c|c|c|c|}
\hline Fatty acid & $\begin{array}{c}\text { Isolate } \\
\text { LM 042 }\end{array}$ & $\begin{array}{c}\text { Catellatospora } \\
\text { tsunoense } \\
\text { IMSNU } 22005^{\mathrm{T}}\end{array}$ & $\begin{array}{l}\text { Catellatospora } \\
\text { citrea } \text { subsp. } \\
\text { citrea } \text { IMSNU } \\
22008^{\mathrm{T}}\end{array}$ & $\begin{array}{l}\text { Catellatospora } \\
\text { citrea } \text { subsp. } \\
\text { methionotrophica } \\
\text { IMSNU } 22006^{\mathrm{T}}\end{array}$ & $\begin{array}{c}\text { Catellatospora } \\
\text { ferruginea } \\
\text { IMSNU 22009 }\end{array}$ & 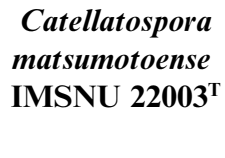 \\
\hline $\mathrm{C}_{12: 0}$ & & & & $1 \cdot 2$ & & \\
\hline $\mathrm{i}-\mathrm{C}_{14: 0}$ & & $1 \cdot 2$ & & $1 \cdot 0$ & $1 \cdot 8$ & \\
\hline $\mathrm{C}_{14: 0}$ & & & & $1 \cdot 4$ & & \\
\hline $\mathrm{i}-\mathrm{C}_{15: 0}$ & $21 \cdot 8$ & $22 \cdot 0$ & $28 \cdot 7$ & $19 \cdot 5$ & $9 \cdot 5$ & $10 \cdot 9$ \\
\hline ai- $C_{15: 0}$ & $3 \cdot 5$ & $4 \cdot 6$ & $3 \cdot 4$ & $4 \cdot 5$ & $21 \cdot 0$ & $3 \cdot 8$ \\
\hline $\mathrm{C}_{15: 0}$ & & & $3 \cdot 0$ & $2 \cdot 1$ & $4 \cdot 9$ & \\
\hline $\mathrm{i}-\mathrm{C}_{16: 1}$ & & & & & & $11 \cdot 7$ \\
\hline $\mathrm{i}-\mathrm{C}_{16: 0}$ & $24 \cdot 6$ & $11 \cdot 8$ & $3 \cdot 1$ & $8 \cdot 3$ & $18 \cdot 3$ & $25 \cdot 3$ \\
\hline$C_{16: 1}$ & $1 \cdot 1$ & $2 \cdot 2$ & & & & \\
\hline $\mathrm{C}_{16: 0}$ & $3 \cdot 6$ & $2 \cdot 2$ & $1 \cdot 9$ & $8 \cdot 1$ & $3 \cdot 1$ & $1 \cdot 9$ \\
\hline $\mathrm{i}-\mathrm{C}_{17: 1}$ & $1 \cdot 5$ & & $3 \cdot 2$ & $1 \cdot 7$ & & $2 \cdot 2$ \\
\hline $\mathrm{i}-\mathrm{C}_{17: 0}$ & $8 \cdot 2$ & $5 \cdot 7$ & $4 \cdot 8$ & $3 \cdot 5$ & $1 \cdot 1$ & 1.9 \\
\hline$C_{17: 1}$ & $16 \cdot 9$ & $18 \cdot 5$ & $16 \cdot 9$ & $13 \cdot 9$ & $15 \cdot 4$ & $12 \cdot 0$ \\
\hline $\mathrm{C}_{17: 0}$ & $1 \cdot 3$ & $8 \cdot 8$ & $14 \cdot 5$ & $9 \cdot 8$ & $20 \cdot 3$ & $1 \cdot 7$ \\
\hline ai- $C_{18: 1}$ & & & & & & $2 \cdot 0$ \\
\hline $10 \mathrm{Me}-\mathrm{C}_{17: 0}$ & & $10 \cdot 4$ & & & & $1 \cdot 8$ \\
\hline $\mathrm{C}_{18: 1}$ & $8 \cdot 5$ & $0 \cdot 6$ & $5 \cdot 2$ & $3 \cdot 1$ & & $12 \cdot 3$ \\
\hline $\mathrm{C}_{18: 0}$ & $6 \cdot 0$ & $4 \cdot 0$ & $4 \cdot 2$ & $13 \cdot 3$ & $4 \cdot 0$ & $4 \cdot 4$ \\
\hline $10 \mathrm{Me}-\mathrm{C}_{18: 0}$ & & $5 \cdot 4$ & & & & $3 \cdot 1$ \\
\hline ai- $C_{19: 0}$ & & & $4 \cdot 7$ & $2 \cdot 4$ & & \\
\hline $\mathrm{C}_{19: 1}$ & & & $2 \cdot 9$ & $1 \cdot 1$ & & \\
\hline $\mathrm{C}_{19: 0}$ & & & $2 \cdot 0$ & $1 \cdot 3$ & & \\
\hline
\end{tabular}


Table 4. Diagnostic physiological characteristics of isolate $\mathrm{LM} 042^{\top}$ and the reference strains of the genus Catellatospora

\begin{tabular}{|c|c|c|c|c|c|c|}
\hline Characteristic & $\begin{array}{c}\text { Strain } \\
\text { LM 042 }^{\mathrm{T}}\end{array}$ & $\begin{array}{c}\text { Catellatospora } \\
\text { tsunoense } \\
\text { IMSNU } 22005^{\mathrm{T}}\end{array}$ & $\begin{array}{c}\text { Catellatospora } \\
\text { citrea subsp. } \\
\text { citrea } \text { IMSNU } \\
22008^{\mathrm{T}}\end{array}$ & $\begin{array}{c}\text { Catellatospora } \\
\text { citrea subsp. } \\
\text { methionotrophica } \\
\text { IMSNU } 22006^{\mathrm{T}}\end{array}$ & $\begin{array}{c}\text { Catellatospora } \\
\text { ferruginea } \\
\text { IMSNU } \\
22009^{\mathrm{T}}\end{array}$ & $\begin{array}{c}\text { Catellatospora } \\
\text { matsumotoense } \\
\text { IMSNU } \\
22003^{\mathrm{T} *}\end{array}$ \\
\hline Growth at $37^{\circ} \mathrm{C}$ & + & - & + & - & + & - \\
\hline \multicolumn{7}{|l|}{ Utilization of carbon source: } \\
\hline D-Arabinose & - & + & - & + & + & - \\
\hline Dextran & - & - & - & - & + & - \\
\hline D-Fructose & + & + & - & - & + & + \\
\hline Melibiose & - & + & + & - & + & - \\
\hline Methyl $\alpha$-D-glucoside & - & - & - & - & + & - \\
\hline D-Raffinose & + & + & - & - & + & + \\
\hline L-Rhamnose & - & + & + & + & + & - \\
\hline D-Ribose & - & - & - & - & + & + \\
\hline D-Trehalose & - & + & + & + & + & + \\
\hline Adonitol & - & - & - & - & + & - \\
\hline D-Mannitol & - & - & - & - & + & - \\
\hline \multicolumn{7}{|l|}{ Growth requirement for: } \\
\hline Thiamin & - & + & - & - & + & - \\
\hline Methionine & - & - & - & + & - & - \\
\hline \multicolumn{7}{|l|}{ Enzyme activity: } \\
\hline Nitrate reductase & + & + & + & + & - & + \\
\hline Urease & - & - & - & - & - & + \\
\hline \multicolumn{7}{|l|}{ Growth on: } \\
\hline $0 \cdot 0001 \%$ Crystal violet & + & - & + & + & + & + \\
\hline $0 \cdot 001 \%$ Brilliant green & + & + & - & - & + & + \\
\hline \multicolumn{7}{|l|}{ Resistance to: } \\
\hline $20 \mu \mathrm{g}$ Vancomycin $\mathrm{ml}^{-1}$ & - & - & - & - & + & + \\
\hline $50 \mu \mathrm{g}$ Novobiocin $\mathrm{ml}^{-1}$ & - & - & - & - & + & + \\
\hline $50 \mu \mathrm{g}$ Gentamicin $\mathrm{ml}^{-1}$ & + & - & - & - & - & - \\
\hline $5 \mu \mathrm{g}$ Neomycin $\mathrm{ml}^{-1}$ & + & + & + & - & - & + \\
\hline $10 \mu \mathrm{g}$ Tetracycline $\mathrm{ml}^{-1}$ & - & - & - & - & + & + \\
\hline
\end{tabular}

* According to Asano et al. (1989) and Lee et al. (1999).

sequence obtained was compared to those of the type strains of the family Micromonosporaceae and related taxa. A phylogenetic tree reconstructed by the neighbour-joining method (Saito \& Nei, 1987) was based on the comparison of 1403 nucleotides present in all strains between bases 34 and 1495 (E. coli numbering system). Our phylogenetic analysis (Fig. 2) indicated that strain LM $042^{\mathrm{T}}$ formed a cluster with members of the genus Catellatospora, which was supported by the bootstrap value of $78 \%$. Strain LM $042^{\mathrm{T}}$ showed the highest $16 \mathrm{~S}$ rDNA similarity values to Catellatospora citrea subsp. citrea $(95 \cdot 0 \%)$, Catellatospora citrea subsp. methionotrophica $(95.2 \%)$ and Catellatospora tsunoense $(94.9 \%)$. We observed an equidistant relationship among strain LM $042^{\mathrm{T}}$, Catellatospora ferruginea and all other members of the Micromonosporaceae genera (levels of similarity 93.0 $94.0 \%)$. DNA-DNA hybridization studies showed that the isolate LM $042^{\mathrm{T}}$ exhibited low levels of DNA relatedness (14-26\%) to the type strains of existing members of the genus Catellatospora.

\section{DISCUSSION}

Comparative 16S rDNA sequence studies provide a rapid and precise way of determining membership of a new strain in a hierarchic classification system of actinomycete bacteria (Stackebrandt et al., 1997). Analysis of the 16S rDNA sequence indicates that isolate LM $042^{\mathrm{T}}$ is phylogenetically related to members of the family Micromonosporaceae within the actinomycete bacteria, which is supported by the presence of an almost complete set of signature nucleotides previously defined for the family Micromonosporaceae (Stackebrandt et al., 1997). The only deviation is the signature at positions 69-122. The sequence of strain LM $042^{\mathrm{T}}$ revealed an A-T pair at these positions, whereas $\mathrm{G}-\mathrm{C}$ pairs are found in those of all other 


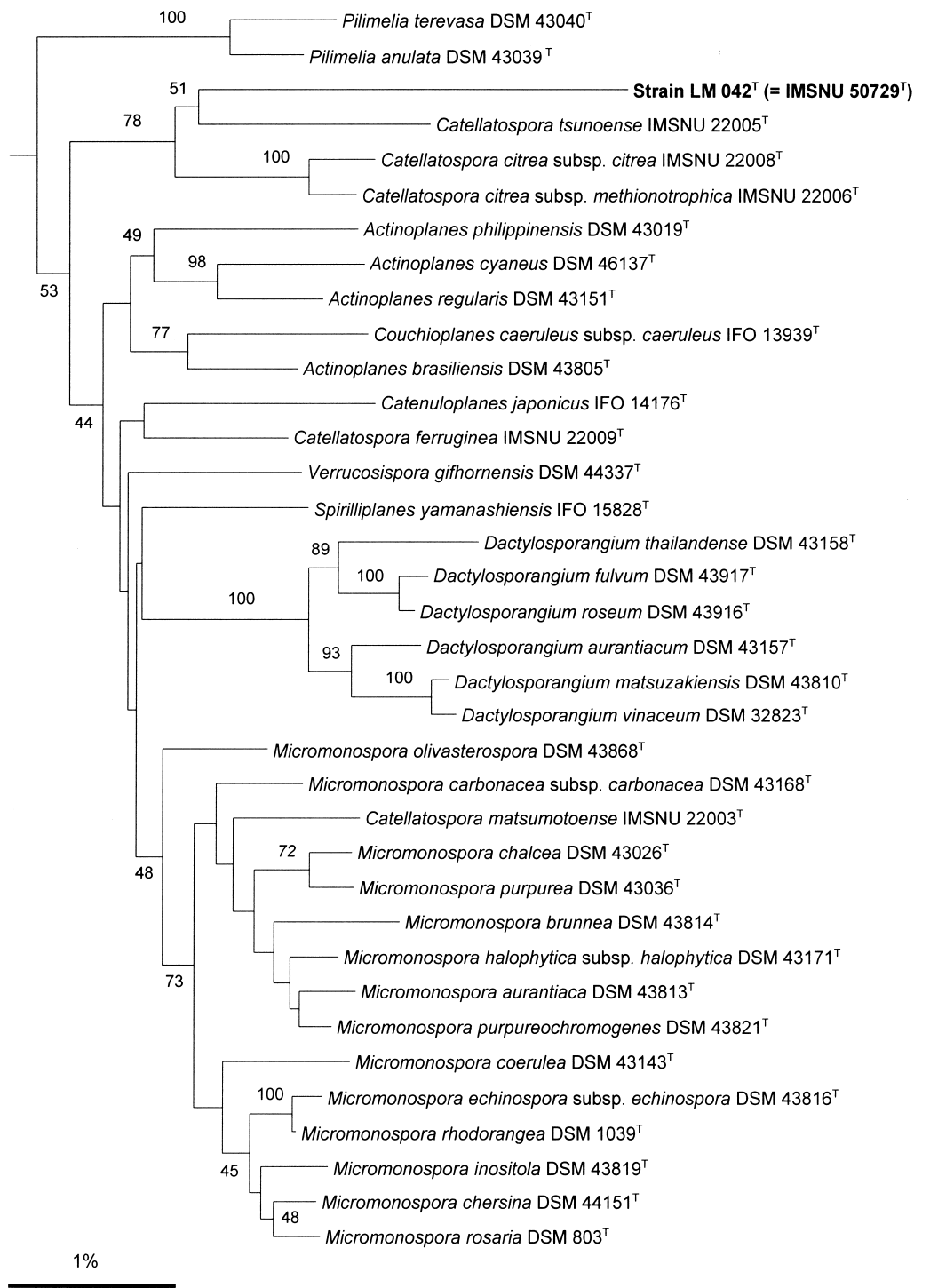

Fig. 2. Phylogenetic tree showing the relationship of isolate $L M 042^{\top}$ to the type strains of the family Micromonosporaceae. The species used as outgroups were Nocardia asteroides, Saccharomonospora viridis, Streptomyces griseus and Streptosporangium roseum. The tree was reconstructed by the neighbour-joining method. The bootstrap values indicated at the branching points are expressed as percentages of 1000 replications. The scale bar indicates 1 nucleotide substitution per 100 nucleotides. members of the family. Therefore, this signature should be excluded from the list of family-specific signatures, along with that at position 1113-1141 previously noted by Rheims et al. (1998). The binary similarity values of the $16 \mathrm{~S}$ rDNA sequences between strain LM $042^{\mathrm{T}}$ and members of the family Micromonosporaceae ranged from 92.9 to $95 \cdot 2 \%, 1 \cdot 2-2 \cdot 7 \%$ lower than the range in which members of this family are related among themselves. The phylogenetic tree shown in Fig. 2 reveals that strain LM $042^{\mathrm{T}}$ forms a cluster with members of the genus Catellatospora. Its phylogenetic neighbours are the two subspecies of the type species, Catellatospora citrea (sequence similarity 95.0-95.2\%), and Catellatospora tsunoense (sequence similarity $94.9 \%$ ). Members of the genus Catellatospora have been previously reported to be phylogenetically heterogeneous (Koch et al., 1996). Catellatospora ferruginea forms a distinct clade within the evolutionary radiation of the family Micromonosporaceae and phylogenetically separates from the cluster encompassing Catellatospora citrea, Catella- tospora tsunoense and isolate LM $042^{\mathrm{T}}(93 \cdot 7-95 \cdot 9 \%$ sequence similarity), and Catellatospora matsumotoense (96.9\% similarity) which is phylogenetically related to members of the genus Micromonospora and members of all other genera of the family Micromonosporaceae $(95 \cdot 8-97.5 \%$ similarity). Catellatospora matsumotoense has been recently proposed to be reclassified as Micromonospora matsumotoense on the basis of its 16S rDNA sequence and phenotypic characters (Lee et al., 1999a).

Isolate LM $042^{\mathrm{T}}$ can be readily distinguished chemically from the type strains of the genus Catellatospora. While strain LM $042^{\mathrm{T}}$ contains mesodiaminopimelic acid, rhamnose, xylose, glucose, mannose and ribose in the whole-cell hydrolysates, all of the Catellatospora reference strains contain 3-hydroxydiaminopimelic acid as an additional diamino acid and arabinose, xylose and galactose as diagnostic sugars (Table 2). Catellatospora ferruginea and Catellatospora matsumotoense also possess 3-O-methylrhamnose as 
an additional characteristic sugar (Asano \& Kawamoto, 1986). The major menaquinone of strain LM $042^{\mathrm{T}}$ is MK-10( $\left.\mathrm{H}_{4}\right)$ (Table 2), whereas other members of the genus contain MK-9 $\left(\mathrm{H}_{4}\right)$ or MK$10\left(\mathrm{H}_{6}, \mathrm{H}_{8}\right)$. The inclusion of the new isolate to the genus Catellatospora increases the heterogeneity of its menaquinone compositions. Considerable quantitative differences are found between isolate LM $042^{\mathrm{T}}$ and members of the genus Catellatospora in cellular fatty acids including the amounts of iso- $\mathrm{C}_{15: 0}$, anteiso$\mathrm{C}_{15: 0}, \mathrm{C}_{15: 0}$, iso- $\mathrm{C}_{16: 1}$, iso- $\mathrm{C}_{16: 0}$, iso- $\mathrm{C}_{17: 0}, \mathrm{C}_{17: 0}, \mathrm{C}_{18: 1}$ and $\mathrm{C}_{18: 0}$. Fatty acids with 19 carbon atoms, which are detected in small amounts in the strains of the type species Catellatospora citrea, are not present in isolate LM $042^{\mathrm{T}}$ and other Catellatospora strains. Finally, low levels of DNA-DNA relatedness $(<26 \%)$ between isolate LM $042^{\mathrm{T}}$ and the type strains of the genus Catellatospora support, along with the chemical characters mentioned above, that this organism should be placed in a new species of the genus Catellatospora.

On the basis of physiological, chemotaxonomic and DNA-DNA hybridization data as well as the uniqueness of its $16 \mathrm{~S}$ rDNA sequence, strain LM $042^{\mathrm{T}}$ is readily differentiated from the previously validated species of the genus Catellatospora, and the name Catellatospora koreensis sp. nov. is proposed.

\section{Description of Catellatospora koreensis sp. nov.}

Catellatospora koreensis [ko.re.en'sis. M.L. adj. koreensis of Korea (pertaining to Korean soil from which the organism was isolated)].

Forms a well-developed, branched substrate mycelium. An aerial mycelium is absent. Short chains of spores are borne directly on substrate mycelium. Spores are cylindrical and non-motile. Spore surface is smooth. The colour of substrate mycelium is cream to light yellow. Globose bodies are formed. A soluble pigment is not produced. Good growth on oatmeal agar, inorganic salt/starch agar, glucose/asparagine agar, glucose/yeast extract agar, Hickey-Tresner agar and Bennett agar. Poor to moderate growth on glycerol/asparagine agar, yeast extract agar, nutrient agar, tyrosine agar and tapwater agar. Aerobic. Grampositive. Catalase-positive. Urease-negative. Nitrate is reduced to nitrite. $\mathrm{H}_{2} \mathrm{~S}$ is not produced. Grows at $\mathrm{pH}$ 6.0-9.0. The temperature range for growth is between 20 and $37^{\circ} \mathrm{C}$. Utilizes L-arabinose, D-cellobiose, Dfructose, D-galactose, D-glucose, D-lactose, maltose, Dmannose, D-raffinose, starch, sucrose and D-xylose as carbon sources. No utilization of D-arabinose, dextran, gluconate, inulin, D-melezitose, melibiose, methyl $\alpha$-Dglucoside, L-rhamnose, D-ribose, salicin, L-sorbose, Dtrehalose, adonitol, dulcitol, butanol, meso-erythritol, ethanol, glycerol, meso-inositol, 2-propanol, propanol, D-mannitol, D-sorbitol and D-xylitol. Does not degrade adenine, hippurate, hypoxanthine, DL-tyrosine and xanthine. Hydrolyses casein, gelatin and starch but not DNA. Grows on $0.001 \%$ brilliant green and $0.0001 \%$ crystal violet. No growth on $3 \% \mathrm{NaCl}$ and $0.01 \%$ lysozyme. Susceptible to $50 \mu \mathrm{g}$ novobiocin $\mathrm{ml}^{-1}, 20 \mu \mathrm{g}$ vancomycin $\mathrm{ml}^{-1}, 100 \mu \mathrm{g}$ streptomycin $\mathrm{ml}^{-1}$ and $10 \mu \mathrm{g}$ tetracycline $\mathrm{ml}^{-1}$, but resistant to $50 \mu \mathrm{g}$ gentamicin $\mathrm{ml}^{-1}$ and $5 \mu \mathrm{g}$ neomycin $\mathrm{ml}^{-1}$. Cell wall contains mesodiaminopimelic acid, rhamnose, ribose, xylose, mannose and glucose (a type II cell wall and a variant of sugar pattern D). The acyl type of muramic acid is glycolyl. The predominant menaquinone is $\mathrm{MK}$ $10\left(\mathrm{H}_{4}\right)$, with minor amounts of $\mathrm{MK}-9\left(\mathrm{H}_{4}\right)$ and MK-10 $\left(\mathrm{H}_{6}\right)$. The diagnostic phospholipids contain phosphatidylethanolamine, diphosphatidylglycerol and phosphatidylinositol. Glucosamine-containing phospholipid and phosphatidylcholine are absent. Mycolic acids are absent. The predominant fatty acids $\left(>15.0 \%\right.$ of total) are iso- $\mathrm{C}_{16: 0}$, iso- $\mathrm{C}_{15: 0}$ and $\mathrm{C}_{17: 1}$ acids. A significant amount of saturated, unsaturated and anteiso-branched fatty acids are also present. The $\mathrm{G}+\mathrm{C}$ content of the DNA is $70.4 \mathrm{~mol} \%$ (determined by HPLC). Isolated from a gold-mine cave. The type strain is LM $042^{\mathrm{T}}\left(=\mathrm{IMSNU} 50729^{\mathrm{T}}\right)$.

\section{ACKNOWLEDGEMENTS}

This work was supported by a research grant for Research Center for Molecular Microbiology, Seoul National University from the Korea Science and Engineering Foundation (KOSEF).

\section{REFERENCES}

Asano, K. \& Kawamoto, I. (1986). Catellatospora, a new genus of the Actinomycetales. Int J Syst Bacteriol 36, 512-517.

Asano, K. \& Kawamoto, I. (1988). Catellatospora citrea subsp. methionotrophica subsp. nov., a methionine-deficient auxotroph of the Actinomycetales. Int $J$ Syst Bacteriol 38, 326-327.

Asano, K., Masunaga, I. \& Kawamoto, I. (1989). Catellatospora matsumotoense $\mathrm{sp}$. nov. and $C$. tsunoense sp. nov., actinomycetes found in woodland soils. Int $J$ Syst Bacteriol 39, 309-313.

Brosius, J., Palmer, J. L., Kennedy, J. P. \& Noller, H. F. (1978). Complete nucleotide sequence of a $16 \mathrm{~S}$ ribosomal RNA gene from Escherichia coli. Proc Natl Acad Sci USA 75, 4801-4805.

Collins, M. D., Goodfellow, M. \& Minnikin, D. E. M. (1982). Polar lipid composition in the classification of Arthrobacter and Microbacterium. FEMS Microbiol Lett 15, 299-302.

Felsenstein, J. (1985). Confidence limits on phylogenies: an approach using the bootstrap. Evolution 39, 783-791.

Felsenstein, J. (1993). PHYLIP (phylogeny inference package), version 3.51c. Seattle, WA: Department of Genetics, University of Washington.

Goodfellow, M., Stanton, L. J., Simpson, K. E. \& Minnikin, D. E. (1990). Numerical and chemical classification of Actinoplanes and some related actinomycetes. J Gen Microbiol 136, 19-36.

Gordon, R. E., Barnett, D. A., Handerhan, J. E. \& Pang, C. H.-N. (1974). Nocardia coeliaca, Nocardia autotrophica, and the nocardia strain. Int J Syst Bacteriol 24, 54-63.

Hopwood, D. A., Bibb, M. J., Chater, K. F. \& 7 other authors (1985). Genetic Manipulation of Streptomyces: a Laboratory Manual. Norwich: The John Innes Foundation.

Jukes, T. H. \& Cantor, C. R. (1969). Evolution of protein molecules. In Mammalian Protein Metabolism, pp. 21-132. Edited by H. N. Munro. New York: Academic Press. 
Koch, C., Kroppenstedt, R. M., Rainey, F. A. \& Stackebrandt, E. (1996). 16S ribosomal DNA analysis of the genera Micromonospora, Actinoplanes, Catellatospora, Catenuloplanes, Couchioplanes, Dactylosporangium, and Pilimelia and emendation of the family Micromonosporaceae. Int J Syst Bacteriol 46, 765-768.

Kroppenstedt, R. M. (1985). Fatty acid and menaquinone analysis of actinomycetes and related organisms. In Chemical Methods in Bacterial Systematics, pp. 173-199. Edited by M. Goodfellow \& D. E. Minnikin. London: Academic Press.

Lechevalier, M. P. \& Lechevalier, H. A. (1970). Chemical composition as a criterion in the classification of aerobic actinomycetes. Int J Syst Bacteriol 20, 435-443.

Lechevalier, M. P., Stern, A. E. \& Lechevalier, H. A. (1981). Phospholipids in the taxonomy of actinomycetes. Zentbl Bakteriol Hyg Abt 1 Suppl 11, 111-116.

Lee, S. D., Goodfellow, M. \& Hah, Y. C. (1999). A phylogenetic analysis of the genus Catellatospora based on 16S ribosomal DNA sequences, including transfer of Catellatospora matsumotoense to the genus Micromonospora as Micromonospora matsumotoense comb. nov. FEMS Microbiol Lett 178, 349-354.

Lee, S. D., Kang, S.-O. \& Hah, Y. C. (2000). Hongia gen. nov., a new genus of the order Actinomycetales. Int J Syst Evol Microbiol 50, 191-199.

Mac Faddin, J. F. (1980). Biochemical Tests for Identification of Medical Bacteria, 2nd edn. Baltimore: Williams \& Wilkins.

Mesbah, M., Premachandran, U. \& Whitman, W. B. (1989). Precise measurement of the $\mathrm{G}+\mathrm{C}$ content of deoxyribonucleic acid by high-performance liquid chromatography. Int J Syst Bacteriol 39, 159-167.

Minnikin, D. E. M. (1988). Isolation and purification of mycobacterial wall lipids. In Bacterial Cell Surface Techniques, pp. 125-135. Edited by I. C. Hancock \& I. R. Poxton. Chichester: Wiley.

Minnikin, D. E., Patel, P. V., Alshamaony, L. \& Goodfellow, M. (1977). Polar lipid composition in the classification of Nocardia and related bacteria. Int J Syst Bacteriol 27, 104-117.

Minnikin, D. E., Hutchinson, I. G., Caldicott, A. B. \& Goodfellow, M. (1980). Thin layer chromatography of methanolysates of mycolic acid-containing bacteria. J Chromatogr 188, 221-233.

Minnikin, D. E., O’Donnell, A. G., Goodfellow, M., Alderson, G., Athalye, M., Schaal, A. \& Parlett, J. H. (1984). An integrated procedure for the extraction of bacterial isoprenoid quinones and polar lipids. J Microbiol Methods 2, 233-241.

Rheims, H., Schumann, P., Rohde, M. \& Stackebrandt, E. (1998). Verrucosispora gifhornensis gen. nov., sp. nov., a new member of the actinobacterial family Micromonosporaceae. Int J Syst Bacteriol 48, 1119-1127.

Saddler, G. S., Tavecchia, P., Lociuro, S., Zanol, M., Colombo, E. \& Selva, E. (1991). Analysis of madurose and other actinomycete whole cell sugars by gas chromatography. J Microbiol Methods 14, 185-191.

Saitou, N. \& Nei, M. (1987). The neighbor-joining method: a new method for reconstructing phylogenetic trees. Mol Biol Evol 4, 406-425.

Shirling, E. B. \& Gottlieb, D. (1966). Methods for characterization of Streptomyces species. Int J Syst Bacteriol 16, 313-340.

Stackebrandt, E., Rainey, F. A. \& Ward-Rainey, N. L. (1997). Proposal for a new hierarchic classification system, Actinobacteria classis nov. Int J Syst Bacteriol 47, 479-491.

Staneck, J. L. \& Roberts, G. D. (1974). Simplified approach to identification of aerobic actinomycetes by thin-layer chromatography. Appl Microbiol 28, 226-231.

Tamura, T., Nakagaito, Y., Nishii, T., Hasegawa, T., Stackebrandt, E. \& Yokota, A. (1994). A new genus of the order Actinomycetales, Couchioplanes gen. nov., with descriptions of Couchioplanes caeruleus (Horan and Brodsky 1986) comb. nov. and Couchioplanes caeruleus subsp. azureus subsp. nov. Int J Syst Bacteriol 44, 193-203.

Tamura, T., Hayakawa, M. \& Hatano, K. (1997). A new genus of the order Actinomycetales, Spirilliplanes gen. nov., with description of Spirilliplanes yamanashiensis sp. nov. Int $J$ Syst Bacteriol 47, 97-102.

Thompson, J. D., Higgins, D. G. \& Gibson, T. J. (1994). CLUSTAL $\mathrm{W}$ : improving the sensitivity of progressive multiple sequence alignment through sequence weighing, position-specific gap penalties and weight matrix choice. Nucleic Acids Res 22, 4673-4680.

Uchida, K. \& Aida, K. (1984). An improved method for the glycolate test for simple identification of the acyl type of bacterial cell walls. J Gen Appl Microbiol 30, 131-134.

Waksman, S. A. (1950). The Actinomycetes: Their Nature, Occurrence, Activities and Importance. Waltham, MA: Chronica Botanica.

Waksman, S. A. (1961). Classification, Identification, and Description of Genera and Species. In The Actinomycetes, vol. 2. Baltimore: Williams \& Wilkins.

Yokota, A., Tamura, T., Hasegawa, T. \& Huang, L. H. (1993). Catenuloplanes japonicus gen. nov., sp. nov., nom. rev., a new genus of the order Actinomycetales. Int J Syst Bacteriol 43, $805-812$. 